\title{
La cartographie hydrologique détaillée et son intérêt pour l'étude des régimes fluviaux
}

\author{
Detailed hydrological mapping \\ and its usefulness in river regime studies
}

\author{
PAR J. TRICART, \\ PHOFESSELIR I LA FACULTÉ DES LETTRES, \\ DIRECTEUR DU GENTRE DE GÉOGRAPHIE APPLIQUÉE (1), UNiVERSTTE DE STRASBOURG \\ ET DES LABORATOIRES DE GÉOGRAPHIE YHYSIQUE ET DE CARTOGHAPHIE
}

\begin{abstract}
Exposé d'une méthode de cartographie hydrologique détaillée des bassins fluviaux mise aut point par le Laboratoire de Géographie physique du Centre de Géographie appliquée de Strasbourg. Cette méthode s'efforce de donier. une image aussi précise que possible des conditions hydrologiques et des phénomènes qui se produisent dans des conditions climatiques revenant auec une fréquence suffisante. L'ntilisation des cartes pour l'analy'se des mécanismes hydrologigues est décrite.
\end{abstract}

\begin{abstract}
A description is given of a detailed hydrological mapping method for river basins which has been developped by the Physical Geography Laboratory of the Strasbourg Applied Geograpy Centre. The aim of this method is to provide the most precise possible picture of the hydrological conditions, and effects experienced under sufficienty frequently recurring climate conditions. The use of these hydrological maps in aralysing hydrological processes is described.
\end{abstract}

Deux méthodes différentes sont utilisées en matière de recherche hydrologique :

- Une méthode statistique, qui exige que la loi des grands nombres soit respectée et qui repose nécessairement sur de grandes séries de mesures. Par suite des liaisons intrinsèques existant entre les facteurs espace et temps, elle s'applique surtout aux grands bassins. Ceux-ci sont hétérogènes mais, du fait même de leur surface, les différences de comportement hydrologique régional se compensent statistiquement. II est alors possible, à partir de longues séries d'observations, d'établir non seulement un régime moyen, mais de prévoir des probabilités

(1) Les travaux dont il est rendu compte dans la présente cornmunication ont été réalisés sous la direction du Professeur J. Tricant, directeur, et de Mlle A. R. Hinsch, collaborateur technique, par diverses équipes des Laboratoires, auxquelles ont notamment participé Mrie I: LE Bourdec, Mlles F. Tourrilhes, L. OTTMaNN, M. VINCENT et M. J. Capolinin. de crues. Des bassins comme ceux du Nil, du Mississipi, ou de moindres dimensions, mais tout aussi complexes, comme celui du Rhin ou du Rhône, se prêtent bien à l'application de cette méthode;

-- Une méthode monographique, qui repose sur des observations plus nombreuses dans l'espace, mais menées pendant des périodes beaucoup plus courtes, et qui vise principalement à analyser les mécanismes de l'écoulement et les modifications de celui-ci en fonction de divers facteurs que l'on s'attache à mesurer. Cette méthode correspond davantage à la forme d'esprit des naturalistes qu'à celle des mathématiciens ou des physiciens, à l'inverse de la première. Elle a été appliquée d'abord à des manifestations particulières de l'écoulement, crues surtout, moindrement étiages. Elle est maintenant à l'origine des bassins expérimentaux. Dans l'étude de ces derniers, on s'efforce de dégager par des analyses fines poursuivies seulement 
pendant quelques années, le comportement d'un bassin considéré comme typique de certaines conditions géographiques.

Bien entendu, les deux méthodes se complètent. Mais, actuellement, par suite de l'orientation d'esprit de la plupart des hydrologues, c'est surtout la méthode statistique qui a bénéficié des efforts de la recherche. Il en résulte une disproportion qui nuit aux applications. Nous sommes beaucoup mieux armés pour faire des prévisions de débits sur un grand fleuve comme la Seine à Paris ou le Rhône à Avignon que dans un petit bassin de 200 ou $500 \mathrm{~km}^{2}$. Or, les exigences de la pratique, accrues par la nécessité dans laquelle nous sommes d'utiliser mieux les ressources en eau, oblige à prévoir des aménagements sur de petits bassins, où, bien souvent, aucune mesure n'a été faite. Tel est le cas pour les installations de lacs collinaires, beaucoup de systèmes d'irrigation, la recharge des nappes souterraines, la lutte contre les crues, etc.

C'est pourquoi, depuis 1958, à la demande principalement, des Services du Génie rural, le Centre de Géographie appliquée s'est attaché à metlre au point des méthodes destinées à faciliter l'étude hydrologique des bassins de petite et moyemne élendue. Elles complètent les analyses de bassins expérimentaux et permettent d'améliorer leurs résultals et de les étendre plus sủrement aux régions voisines. Elles reposent sur l'établissement d'un document de base, qui est la carte hydrologique détaillée, dont nous allons définir la conception, puis dont nous étudierons la réalisation et l'utilisation.

\section{Conception de la carte hydrologique détaillée :}

L'objet de la carte est de présenter une image précise et exacte des conditions offertes à l'écoulement d'un espace déterminé. Elle permet, par planimétrage, de déterminer les surfaces affectées par tel ou tel facteur ou caractérisées par telle ou telle combinaison de facteurs aboutissant à définir un type de comportement hydrologique. La carte constitue ainsi un instrument indispensable pour l'interprétation des mesures hydrologiques.

Prenons un exemple banal, celui du calcul des coefficients de ruissellement: habituellement, on le calcule comme si tout le bassin avait joué de la même manière. Or, lors des averses, on observe, sur le terrain :

- des surfaces où les gouttes se contentent de mouiller la surface du sol et s'infiltrent;

- des surfaces où se forment de petites mares pelliculaires, avec, sur les pentes, écoulement d'un film d'eau qui s'infiltre au bout de quelques décimètres, de quelques mètres au plus. C'est l'écoulement aréolaire, qui passe à un écoulement discontinu;

- lécoulement discontinu, lequel est formé de filets peu puissants qui butent contre les obstacles et se divisent, par exemple, contre les touffes de végétation, les pierres, ou des embâcles de feuilles mortes et de brindilles ou qui s'étalent sur des plages plus unies. Dans les deux cas, une bonne partie de l'eau, souvent la totalité, s'infiltre. Il ne s'agit donc que d'une infiltration différée.

- l'écoulement concentré élémentaire, enfin, qui marque l'apparition d'un écoulement hiérarchisé en réseau. Se reproduisant avec une certaine fréquence (à peu près annuelle), il inscrit sa marque dans le sol sous la forme d'un talweg, bien modeste d'ailleurs et qui, dans les champs, est effacé par le labour. Il y a là une différence essentielle avec l'écoulement discontinu qui est instable et se réorganise à chaque averse en fonction d'obstacles changeants, ne pouvant ainsi inscrire une marque durable dans la microtopographie.

Suivant l'intensité des averses, l'état du sol (plus ou moins perméable) celui du couvert végétal (arbres avec ou sans feuilles), les divers types d'écoulement ont une plus ou moins grande extension. Une averse violente, un sol imperméabilisé par la sécheresse, ou désagrégé par le gel, surtout à une saison où la végétation est dépouillée, étendent le ruissellement concentré élémentaire au détriment des autres formes La topographie intervient également, bien entendu : une pente forte, qui permet au ruissellement discontinu de vaincre les obstacles et, ainsi de devenir continu, facilite la réalisation d'un écoulement concentré. On aboutit ainsi à une notion extrêmement importante, celle de la surface-seuil d'écoulement concentré. Elle correspond à la surface minimale à l'issue de laquelle apparaît un ruisselet qui s'intègre dans un réseau hydrographique organisé. Bien entendu, la notion est inséparable d'une donnée de fréquence. Lors d'une crue très violente, comme celle de juin 1957 dans le Queyras, la surfaceseuil d'écoulement concentré a été diminuée en moyenne de plus de moitié. Le nombre des talwegs élémentaires (de $1^{\mathrm{er}}$ ordre, ceux qui ne recoivent aucun affluent), a été approximativement doublé. Cela s'est traduit par la formation de ravines sur les versants. Il est à noter que les travaux de G. Wolman et L. B. Leopold [1] assignent également une fréquence grossièrement annuelle à l'écoulement à pleins bords qui faconne les lits des cours d'eau plus importants. Nous saisissons donc là un critère de la crue annuelle, dont l'intérêt hydrologique est bien connu. 
Ces observations montrent que le calcul d'un coefficient de ruissellement effectué sur toute la surface d'un bassin n'a guère de sens en dehors des régions de bad-lands, les seules, où le ruissellement est généralise. Dans tous les autres cas, on aboutit à une sous-estimation, notamment dans les régions peu accidentées où les surfaces effectivement affectées par l'écoulement concentré peuvent s'abaisser à $10 \%$ du total (certaines parties des Aspres du Roussillon) ou même à moins de $1 \%$ (Champagne crayeuse). Or, dans les secteurs qui ne participent pas à l'écoulement concentré, la fraction des précipitations qui n'est pas restituée à l'atmosphère par évapo-transpiration est entièrement disponible pour l'alimentation des nappes phréatiques, écoulement hypodermique compris.

Seule une connaissance précise, acquise sur le terrain, de semblables faits peut permettre des calculs plus précis sur le ruissellement, l'alimentation des nappes, des débits d'étiage.

L'objet de nos cartes est de fournir de telles données, planimétrables. Nous laissons de côté données climatiques et débits trop variables, que l'on représente à part sur des cartes potamologiques fondées sur les moyennes ou figurant une situation particulière. Nous ne retenons que les conditions de l'écoulement, potentiel permanent sur lequel jouent les facteurs climatiques plus changeants :

- caractéristiques des terrains, essentiellement les conditions de filtration et de rétention, qui jouent en sens inverse;

- pente des versants et interflures;

- grands types de couvertures végétales.

Mais la carte ne doit pas se limiter à fournir les facteurs de l'écoulement, elle doit aussi représenter ses formes et ses caractéristiques:

- les types d'écoulement discontinu et concentré en fonction des traces qu'il imprime dans le micromodelé, ce qui est un bon critère de fréquence;

- les régimes d'écoulement concentré : sporadique, saisonnier et pérenne;

- les zones inondables en crue annuelle ou décennale;

- les caractères des lits qui influent sur l'écoulement : calibrés, irréguliers, encombrés de blocs ou de végétation, parsemés de bancs alluviaux, canalisés, etc.;

Enfin, la carte doit renseigner sur les phénomènes géomorphologiques intéressant les cours d'eau et faire apparaître :

- les ravinements et les principales sources de débit solide;

- les berges sapées et les secteurs de creusement;
- les zones d'accumulation, banes d'alluvions, cônes de déjections, etc., avec indication de leur nature.

Ainsi conçue, la carte hydrologique détaillée permet une exploitalion précise des mesures hydrologiques et une analyse des mécanismes de l'écoulement et des transports solides. Elle répond aux exigences tant de la recherche fondamentale que de la recherche appliquée.

\section{Réalisation de la carte (2):}

Le nombre et la variété des données à figurer a posé un problème technique de cartographie ardu. Il a fallu faire un choix entre les données les plus importantes, portées sur la carte ellemême, et les données connexes, figurant sur des calques ou des cartons.

a) En fond de carte, sont figurées les caractéristiques hydrologiques des terrains qui forment les versants et interfluves.

Les données essentielles à figurer sont la filtration et la rétention, tout en tenant comple des différences de comportement éventuelles des formations superficielles et du substratum. Le mode de représentation doit être assez souple pour s'appliquer aussi bien aux régions où de nombreuses mesures ont été faites (par exemple, périmètres à irriguer) qu'à d'autres où seul est possible un examen qualitatif sur le terrain.

Nous arons décidé de figurer en teinte plate les argiles, car leurs caractéristiques et les problèmes qu'elles posent sont très particuliers. Leur vitesse de filtration est très lente et leur capacité de rétention est très élevée, mais elles ne restituent que très peu de l'eau retenue. A cause de cela, leur épaisseur importe peu : nous nous sommes contentés de fixer un seuil pour leur figuration (épaisseur minimale de $0,5 \mathrm{~m}$ ). Les schistes argileux compacts, dont le comportement diffère peu de celui des argiles, sont indiqués au moyen de la même teinte en trame serrée.

Pour les autres terrains, nous avons, après divers essais, représenté les superpositions au moyen de bandes verticales alternées dont la valeur indique l'épaisseur des formalions superficielles : 0,2 à $0,5 \mathrm{~m}, 0,5$ à $3 \mathrm{~m}$, et plus de $3 \mathrm{~m}$.

Les catégories représentées sont définies essentiellement par la lithologie et leurs caractéristiques peuvent ainsi être précisées au moyen de mesures effectuées soit sur le terrain, soit au laboratoire.

(2) Le contenu de la carte et certaines des techniques do réalisation ont été mises au point au cours de séances de travail au Service Technicue Central Hydraulique du Génie Rural avec MM. Dalloot, Hlaver et LecanPFNTIEI. 
Pour les formations meubles (y compris les formations superficielles), nous avons retenu les catégories habituellement utilisées par les pédologues, dont les travaux peuvent ainsi être mis à profit et vice-versa :

- argileux,

- argilo-sablo-caillouteux,

- argilo-sableux,

- sableux,

- sablo-caillouteux,

- croûtes compactes (alios, croûtes calcaires quaternaires des régions méditerranéennes, etc.),

- limons (loess notamment).

Pour les formations cohérentes, qui constituent généralement le substratum, sauf dans le cas d'affleurements de roche nue, nous avons regroupé les types de roches en grandes catégories ayant un comportement de même nature. Chacune est représentée par un certain figurétype dans lequel des variantes sont introduites en cas de besoin pour représenter des nuances importantes. Nos types sont les suivants :

- Roches finement poreuses, circulation lente et généralisée, nappe phréatique continue (craie, grès et conglomerats peu compacts, etc.);

- Roches faiblement poreuses, mais fissurées, à circulation diaclasique rapide, mais possibilités de nappes phréatiques continues (calcaires en bancs minces, grès diaclasés ou en bancs minces, marno-calcaires faiblement marneux, etc.);

- Roches compactes, à porosité très faible ou nulle, mais parcourues de diaclases permettant une circulation localisée (calcaires en bancs massifs, glès massifs, basaltes, certains quartzites fissurés, certains granites ou gneiss écrasés, etc.);

- Roches compactes, à porosité très faible ou nulle, mais massives, pratiquement sans circulation diaclasique (schistes compacts, granites ou gneiss massifs, certains conglomérats, etc.).

Les types de superpositions permettent d'apprécier les possibilités d'écoulement hypodermique et de nappes superficielles. Par exemple, en Vendée, les arènes tertiaires recouvrant les gneiss ou les granites donnent toujours une nappe superficielle qui persiste une partie de l'été et alimente des sources là où la reprise d'érosion quaternaire amène les vallons à s'inciser dans le substratum cristallin. Sur les schistes briovériens, ou la circulation diaclasique est à peu près nulle, les altérites sont plus argileu- ses, de sorte que la filtration y est beaucoup plus lente. L'efret modérateur sur les crues est moindre, les sourcins sont moins abondants, mais larissent plus lentement l'été.

b) En surchargc, sont représentés les phénomènes hydrologiques.

Tout ce qui est lié à la circulation souterraine est figuré en rouge, ce qui le fait apparaître nettement. Les dolines, avens, pertes, apparaissent sous la forme de points rouges de divers types. Les sources et résurgences sont également portées avec des signes spéciaux permettant d'indiquer le débit. Une partie de ces données figure déjà normalement sur les cartes hydrogéologiques, mais il est nécessaire qu'elles apparaissent aussi sur une carte hydrologique. Cela suppose seulement une coordination lors des levés, coordination qui s'impose d'ailleurs pour toute une série de raisons.

Tout ce qui est lié à la circulation superficielle est représenté en noir : zones de ruissellement diffus, tous les talwegs. Pour ces derniers, il est tenu compte de leur largeur (au moyen de sa continuité) et des caractéristiques du lit (rocheux, à fond mobile, à berges nettes ou floues, à bancs de galets, calibré ou non, avec rapides ou chutes, etc.).

Les zones inondables sont indiquées au moyen d'une teinte spéciale qui sert à figurer les formations alluviales du fond de vallée ou qui vient en surcharge sur le figuré de la roche en place lorsque le cas se présente. De la sorte, on peut reconnaître si les crues alimentent des nappes phréatiques d'alluvions ou non. Un signe particulier est réservé aux tourbières, un autre aux marais. Les secteurs où les crues s'étalent et s'écrêtent, ceux où elles sont canalisées entre les versants rapprochés sont ainsi mis en évidence.

c) Sur des calques, apparaissent des données complémentaires qu'il n'est pas possible ou pas souhaitable de figurer sur la carte elle-mème. A défaut de calques, on peut également utiliser d'autres cartes, établies à la mềme échelle et superposables sur une table lumineuse.

Sont à confronter avec la carte hydrologique : - la carte hydrogéologique;

- la carte morphométrique. Sur la carte hydrologique sont reportées les courbes de niveau de la carte topographique, dont le bassin est renforcé afin de les rendre plus visibles. Cependant, il ne s'agit que de données brutes, qui ne sont pas suffisantes pour une étude hydrologique. Aussi avons-nous décidé de dresser des cartes de la valeur des pentes comportant également indication des lignes principales de rupture de pente, notamment des lignes de partage des eaux. Ces 
dernières figurent d'ailleurs sur la carte hydrologique. De la sorte, par planimétrage, il est possible de calculer la proportion d'un bassin constitué par des pentes fortes ou faibles, de dresser des courbes cumulatives des pourcentages de surfaces occupées par les diverses classes de pentes en amont d'un point donné, de calculer les distances auxquelles se trouvent les divers secteurs de pentes raides donnant un écoulement torrentiel, etc. Les pentes des talwegs sont également portées sur ces cartes. Des données précieuses sont ainsi fournies pour l'étude des vitesses de concentration des flots de crues;

- la carte des types de végétation, car ceux-ci jouent un très grand rôle aussi bien dans l'érosion que dans les conditions de l'écoulement. Il ne s'agit pas d'une carte géobotanique, complexe et longue à établir, mais d'une carte simplifiée, quoique plus complète que les simples cartes topographiques. Nous avons prévu les catégories suivantes:

- surfaces nues (rochers, etc.);

- forêts;

- landes et broussailles;

- surfaces occupées en permanence par l'herbe (prés, pâtures);

- surfaces faisant l'objet de façons agricoles (labours, jardins, vignes, vergers desherbés).

Cette carte est dressée essentiellement d'après les photographies aériennes avec contrôle sur le terrain. Elle se prête, elle aussi, à des planimétrages. Comme elle est peu chargée, elle peut servir à portex les données climatiques dont on dispose : isohyètes et courbes d'égale évapotranspiration. Mais bien souvent, les données sont insuffisantes pour être traitées au $1 / 50000^{\circ}$ et on doit se contenter de carton à petite échelle.

La conception cartographique que nous venons d'exposer et les techniques que nous avons adoptées pour la réaliser imposent des impératifs d'échelle. Nos essais, à la demande du Génie rural, ont été réalisés les uns au $1 / 20000^{\circ}$ (Pyrénées orientales), les autres au $1 / 50000^{\mathrm{e}}$. Cette dernière échelle permet de figurer avec une bonne précision le comportement hydrologique des interfluves et les données d'liydrologie souterraine. Elle est un peu petite pour les caractéristiques des talwegs qui sont mieux rendues au $1 / 20000^{\circ}$. En dehors de ce dernier point, il n'y a aucune difficulté à utiliser les mêmes méthodes pour un $100000^{\circ}$. A des échelles plus petites, une systématisation très poussée devient nécessaire et les avantages présentés par la méthode cartographique décroissent. Par contre, aucune difficulté ne s'oppose à la rédaction de cartes à plus grande échelle, au $1 / 10000^{\mathrm{e}}$ ou au $1 / 5000^{\mathrm{e}}$, par exemple, pour l'étude de petits bassins expérimentaux. Il est possible, au contraire, d'y porter davantage de détails et d'atteindre une plus grande précision.

\section{L'utilité des cartes hydrologiques détail- lées :}

Les cartes hydrologiques ne remplacent aucune des techniques actuellement en usage. Elles les complètent seulement et leur permettent de donner de meilleurs résultats.

Par exemple, elles aident les hydrogéologues à résoudre les problèmes d'alimentation des nappes. Nos cartes montrent nettement l'extension des régions à ruissellement et de celles sans écoulement superficiel régulier, donc où l'excédent des précipitations sur l'évapotranspiration s'infiltre. Il est aisé de calculer ce que ces dernières fournissent aux nappes. Nos essais du Roussillon ont été faits à la demande du C.E.R.G.A. dans ce but. De même, les masses d'eau qui circulent lentement dans les alluvions poreuses sont plus efficaces pour alimenter les nappes que l'infiltralion directe d'interfluve. Par exemple, nous pensons que les nappes artésiennes des calcaires jurassicues du centre du bassin de Paris doivent plus à l'infiltration dans le fond des vallées de la Meuse, et de l'Aire qu'à l'infiltration directe sur les plateaux calcaires, trop secs, où l'excédent de la pluviosité sur l'évapotranspiration est médiocre.

Nos cartes ont surtout, cependant, pour objet, d'aider à mieux comprendre et à mesturer plus exactement les phénomènes hydrologiques. Dans les bassins pour lesquels on dispose déjà de données, elles permettent de les commenter d'une manière plus poussée et de préciser davantage les mécanismes. Par exemple, dans le bassin de la Vendée où n'existent que quelques évaluations de débits, elles permettent de comprendre une incroyable torrentialité, malgré un régime océanique et un faible relief. Presque partout, l'infiltration est faible et les conditions sont favorables à un écoulement brutal et massif en cas de pluies dépassant $20 \mathrm{~mm}$ en 24: h. Les interfluves ont toujours des pentes supérieures à $1^{\circ}$, ce qui, sur les argiles kaoliniques tertiaires d'altération des schistes, permet un ruissellement qui devient assez rapide dès que la pente dépasse $2-3^{\circ}$. Les versants proches des cours d'eau sont formés par les schistes imperméables, à peine masqués par une pellicule superficielle d'argile pierreuse dont la capacilé de rétention est quasi nulle. Icurs pentes de 10-20" permettent un ruissellement intense et rapide. Enfin, la disposition des zones de ruissellement maximal est telle que les ondes de crue se super- 
posent dans la basse vallée, aux barrages de Mervent. Un très léger décalage, ne dépassant pas $1 \mathrm{~h}$, provient d'un étalement un peu plus important des crues dans la vallée de la Vendée supérieure.

Les cartes font ressortir des types de bassins et permettent de mieux choisir les sites de stations de jaugeage. Elles facilitent l'extrapolation de leurs résultats aux régions voisines. Les planimétrages, les analyses morphométriques fondées sur la carte permettent de comparer avec précision des bassins voisins et, connaissant des réactions hydrologiques de l'un d'entre eux, de prévoir celles des autres. Elles sont un complément de la méthode des bassins expérimentaux et permettront de mieux saisir les phénomènes naturels. Elles servent aussi directement à l'étude des aménagements en fournissant des données sur l'instabilité des lits, l'extension des inondations, la provenance des matériaux transportés.

\section{RÉFÉRENCE}

[1] Wolman (G.) et Leopold (L.B.), 1957 : River flood plains : some observations on their formation. I.S. Géol. Snrvey, Prof. Paper 282, C, p. 85-109.

\section{COMMENTAIRE}

President : M. BECKER

M. le Président se félicite d'aroir entendu cette frés brillante conférence qui a donmé des aperçus pouvant, pour l'avenir, devenir très féconds daris les méthodes de recherehes et d'investigation.

H. le Présitent remercie, au nom de l'Assembléc, M. le professeur Tricart de son exposé.

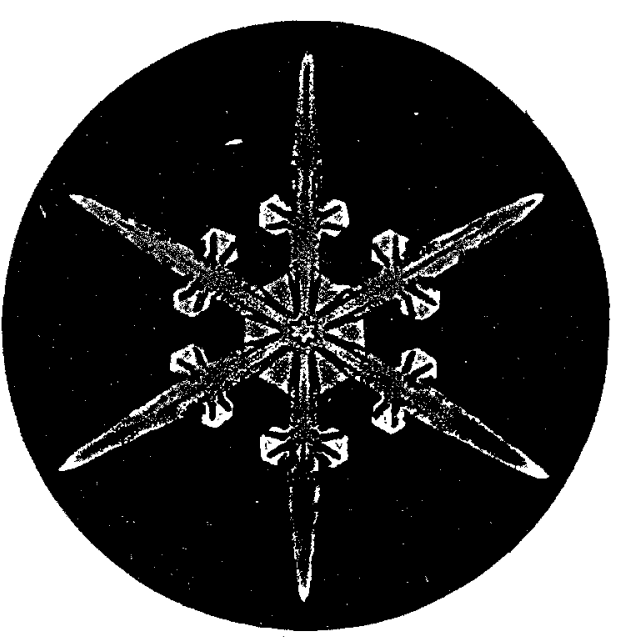

